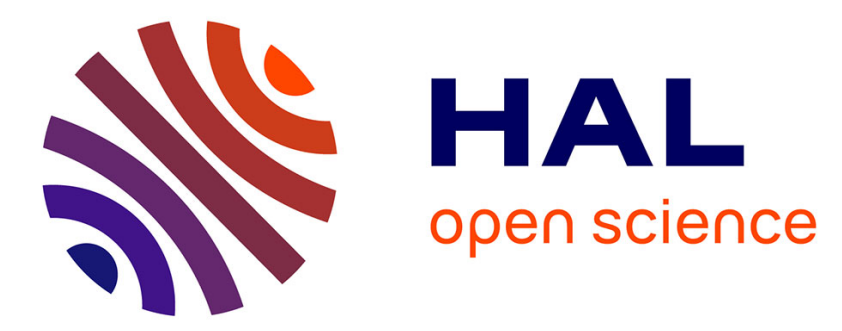

\title{
Carbon membranes for efficient water-ethanol separation
} Simon Gravelle, Hiroaki Yoshida, Laurent Joly, Christophe Ybert, Lydéric Bocquet

\section{To cite this version:}

Simon Gravelle, Hiroaki Yoshida, Laurent Joly, Christophe Ybert, Lydéric Bocquet. Carbon membranes for efficient water-ethanol separation. Journal of Chemical Physics, 2016, 145 (12), pp.124708 - 124708. 10.1063/1.4963098. hal-01628789

\section{HAL Id: hal-01628789 \\ https://hal.science/hal-01628789}

Submitted on 9 Dec 2017

HAL is a multi-disciplinary open access archive for the deposit and dissemination of scientific research documents, whether they are published or not. The documents may come from teaching and research institutions in France or abroad, or from public or private research centers.
L'archive ouverte pluridisciplinaire HAL, est destinée au dépôt et à la diffusion de documents scientifiques de niveau recherche, publiés ou non, émanant des établissements d'enseignement et de recherche français ou étrangers, des laboratoires publics ou privés. 


\title{
Carbon membranes for efficient water-ethanol separation
}

\author{
Simon Gravelle, ${ }^{1}$ Hiroaki Yoshida, ${ }^{2,3}$ Laurent Joly, ${ }^{1}$ Christophe Ybert, ${ }^{1}$ \\ and Lydéric Bocquet, ${ }^{2, a)}$ \\ ${ }^{1}$ Institut Lumière Matière, UMR5306 Université Lyon 1-CNRS, Université de Lyon, 69622 Villeurbanne, France \\ ${ }^{2}$ Laboratoire de Physique Statistique, Ecole Normale Supérieure, UMR CNRS 8550, PSL Research University, \\ 24 rue Lhomond, 75005 Paris, France \\ ${ }^{3}$ Toyota Central RED Laboratories, Inc., Nagakute, Aichi 480-1192, Japan
}

(Received 5 July 2016; accepted 7 September 2016; published online 27 September 2016)

\begin{abstract}
We demonstrate, on the basis of molecular dynamics simulations, the possibility of an efficient water-ethanol separation using nanoporous carbon membranes, namely, carbon nanotube membranes, nanoporous graphene sheets, and multilayer graphene membranes. While these carbon membranes are in general permeable to both pure liquids, they exhibit a counter-intuitive "self-semi-permeability" to water in the presence of water-ethanol mixtures. This originates in a preferred ethanol adsorption in nanoconfinement that prevents water molecules from entering the carbon nanopores. An osmotic pressure is accordingly expressed across the carbon membranes for the water-ethanol mixture, which agrees with the classic van't Hoff type expression. This suggests a robust and versatile membrane-based separation, built on a pressure-driven reverse-osmosis process across these carbon-based membranes. In particular, the recent development of large-scale "grapheneoxide" like membranes then opens an avenue for a versatile and efficient ethanol dehydration using this separation process, with possible application for bio-ethanol fabrication. Published by AIP Publishing. [http://dx.doi.org/10.1063/1.4963098]
\end{abstract}

\section{INTRODUCTION}

Ethanol is the most commonly used commercial biofuel, promising environmental and economic benefits, such as a reduction of consumption of crude oil and related environmental pollution. ${ }^{1,2}$ However, the fabrication of bioethanol requires an unavoidable step: ethanol dehydration. ${ }^{3}$ The separation of ethanol from water is commonly performed by heating processes, e.g., pervaporation or distillation, and this step represents the bulk of the cost for the production of ethanol from biomass. ${ }^{4}$ A reduction of the cost of ethanol dehydration is thus critical regarding the global production of bio-ethanol which reached 46 billion liters in 2007 and could grow up to 125 billion liters by $2020{ }^{1}$

Numerous solutions exist for the dehydration of ethanol, for instance, ordinary distillation, azeotropic distillation, extractive distillation (with liquid solvent or with dissolved salt), liquid-liquid extraction-fermentation hybrid, adsorption, and membrane separation. ${ }^{3}$ Currently, pervaporation, which consists in the partial vaporization of the liquid through a membrane, is considered as one of the most effective and energy-saving processes for the separation of ethanol and water. ${ }^{5-7}$ However, this method requires to heat the system up to $\sim 80^{\circ} \mathrm{C}$ for water-ethanol separation and, just as every thermal separation method, suffers from the disadvantage of a high energy penalty, associated with heat losses to the environment, heat losses due to minimal driving forces, and losses due to boiling point elevation. ${ }^{8}$

On the other hand, membrane-based separation methods, such as ultra-filtration and reverse osmosis (RO), have

\footnotetext{
a)Electronic mail: lyderic.bocquet@ens.fr
}

gained considerable importance because they offer superior treatment at modest cost, high stability and efficiency, and low energy requirement. ${ }^{9,10}$ Particularly, RO is currently the most important desalination technology ${ }^{11}$ thanks to a very low cost in comparison with thermal desalination technology. ${ }^{12}$ The membrane separation is thus seen as a viable and effective technology at both laboratory and industrial scales. However, the applicability of the membrane separation is not obvious when it comes to two species that are neutral and have very similar size, such as ethanol and water.

In this paper, we demonstrate, using molecular dynamics (MD) simulations, that water-ethanol separation can be achieved with carbon-based membranes. We show that in the presence of water-ethanol mixtures, nanoporous carbon membranes may become fully impermeable to water while keeping a high permeability to ethanol. This is in spite of these carbon membranes being in general permeable to both water and ethanol when they are used as pure components. ${ }^{13}$ In the following, we coin accordingly this behavior "self-semi-permeability" to highlight the change of the membrane permeability in the presence of mixtures, which occurs without any further external action. This counterintuitive result is highlighted by the existence of an osmotic pressure for the ethanol-water mixture across the membrane, which has to be bypassed in order to separate ethanol from water. The basic mechanism for this specific separation lies in a preferred adsorption of ethanol as compared to water. We found a similar separation property with three different types of carbon based membranes: namely, carbon nanotubes (CNTs); a single graphene sheet pierced with nanopores; and a multilayer graphene membrane, mimicking the porous 
structure of reduced graphene-oxide (GO) membranes. This highlights the robustness and versatility of the underlying mechanism.

Our results suggest an efficient membrane-based method for the separation of water from ethanol. Thanks to the recent progress made for the development of GO membranes, we believe that this versatile method may offer a new solution for ethanol dehydration, with a significant potential impact on the production of bio-ethanol.

\section{ETHANOL-WATER MIXTURES ACROSS CARBON MEMBRANES: MD SIMULATIONS}

We investigate the hydrodynamic permeability of different carbon-based membranes to ethanol-water mixtures using MD simulations, employing the open source code LAMMPS. $^{14,15}$ The system consists of two reservoirs separated by a carbon-based membrane, see Fig. 1(a). The top reservoir initially contains pure ethanol while the bottom one is filled with a mixture of water and ethanol. Periodic boundary conditions are imposed in all directions, and two graphene sheets at the top and bottom ends of the reservoir are used as a piston to control the pressure in each reservoir.

We consider three membranes with different porous structures: First, a carbon nanotube (CNT) membrane, consisting of two pierced graphene sheets connected by short CNTs (length $L=13 \AA$ and radius $a_{c}$ varied between 3.5 and $6.2 \AA$, see Figs. 1(b) and 1(e)). We also modeled a nanoporous graphene membrane, using a single graphene sheet pierced with nanometric circular pores with radius $a_{c}$ varied between 3.5 and $6.2 \AA$ (Fig. 1(c)). Finally, we consider a multilayer graphene membrane, made of stacked graphene sheets, pierced with nanoslits of width $D=14.1 \AA$ (Figs. 1(d) and 1(f)). The nanoslits are arranged in a staggered fashion with offset $L=34.1 \AA$, forming highly ordered films with 2D nanochannels between the sheets. The inter-layer distance of this membrane, denoted by $h_{c}$, is varied from 6.8 to $20 \AA$. This porous structure, consisting of a "millefeuille" of pure graphene sheets, is considered as a simplified model for GO membranes. This corresponds merely to "reduced" GO membranes, for which the chemical groups covering the graphene sheets can be eliminated.

For the interaction potentials, we employ the TIP4P/2005 water model. ${ }^{16}$ The ethanol molecule is described with the united atom model optimized potentials for liquid simulations (OPLS-UA). ${ }^{17,18}$ The parameters for the carbon atoms of the wall are extracted from the AMBER96 force field, ${ }^{19}$ and the Lorentz-Berthelot mixing rules are used to determine the Lennard-Jones parameters for the cross-interactions. Finally, the positions of carbon atoms in the membrane are fixed and the graphene pistons move as a rigid body. Note that simulations with flexible and fixed walls have been shown to give similar results for the statics and friction of confined liquids. ${ }^{20-22}$

During simulation runs, the system is maintained at $300 \mathrm{~K}$ using two Berendsen thermostats, one in each reservoir. ${ }^{23}$ Those thermostats are applied to molecules at more than $5 \AA$ from the membrane so that the flow in the membrane and at the membrane entrances is not affected by the thermostating procedure. ${ }^{24}$ The pressures of top and bottom reservoirs are maintained at $p_{0}=1$ bar and $p_{0}+\Delta p$, respectively. After the equilibration for at least $0.1 \mathrm{~ns}$ with a plug preventing the exchange of molecules across the membrane, the time evolution of the number of molecules in the reservoirs is recorded.

\section{RESULTS}

As quoted above, we have explored filtration across three types of carbon membranes: CNT membranes, graphene membranes pierced with nanopores, and multilayer graphene membranes, mimicking GO membranes. ${ }^{26}$ In the following, we start by investigating CNT membranes and then the results are generalized to the two other types of carbon membranes. We anticipate that similar results are obtained for the various types of membranes. (a)

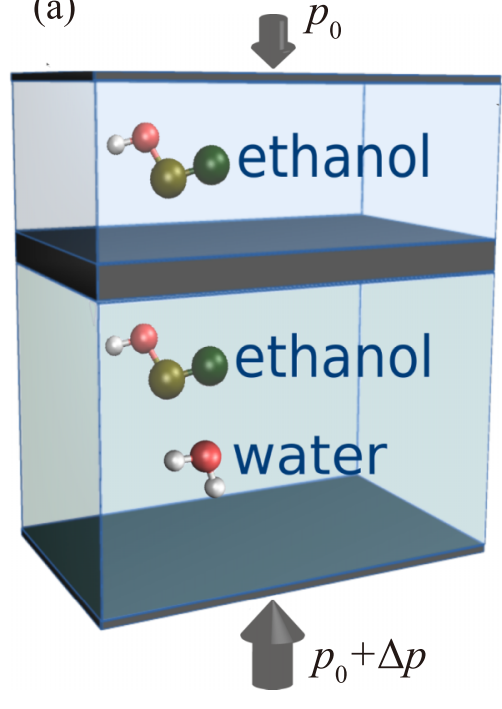

(b)

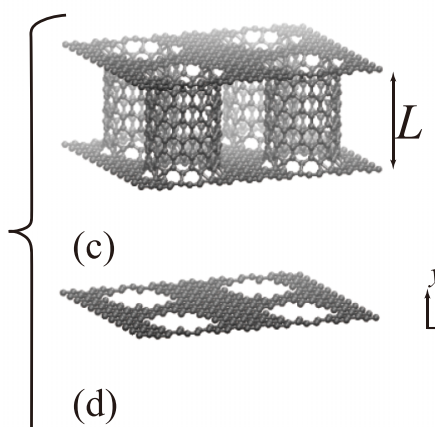

(e)

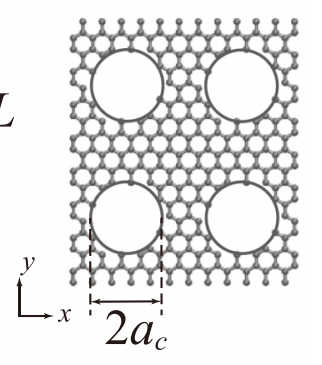

(f)

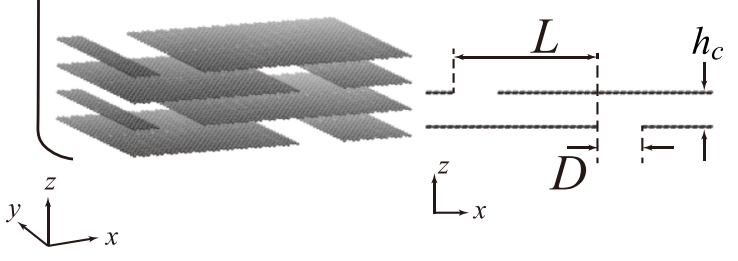

FIG. 1. (a) Schematic of the system. (b) Carbon nanotube membrane. (c) Membrane of single pierced graphene sheet. (d) Multilayer graphene membrane. (e) Top view of panel (b). (f) Side view of panel (d). 
(a)

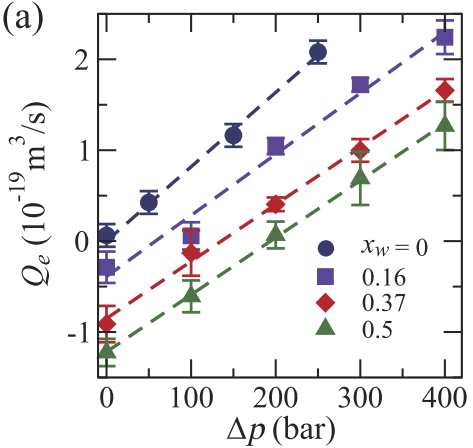

(b)

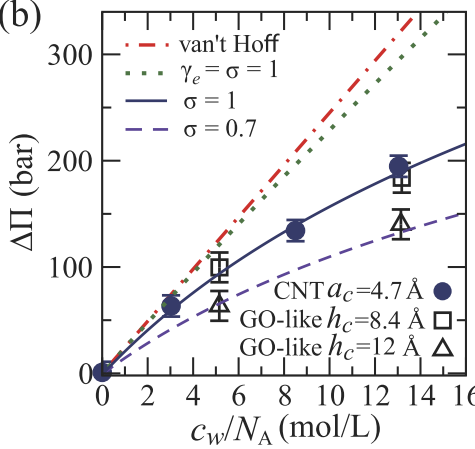

(c)

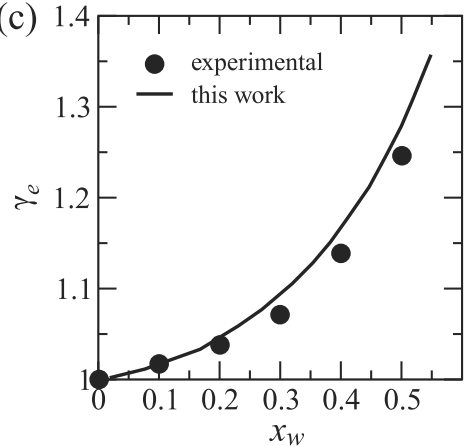

FIG. 2. (a) Flow rate of ethanol $Q_{e}$ per tube in a CNT membrane with $a_{c}=4.7 \AA$ as a function of the applied pressure difference $\Delta p$ for various initial molar fractions of water $x_{w}$. The dashed lines are linear fit, whose slope is the hydrodynamic permeance. (b) Osmotic pressure $\Delta \Pi$ as a function of the initial concentration of water $c_{w}$ for a CNT membrane and GO-like membranes. The prediction of Eq. (5) with fitted values for the activity coefficient $\gamma_{e}$ is shown by the solid line $(\sigma=1)$ and the dashed line $(\sigma=0.7)$, and that with assuming $\gamma_{e}=\sigma=1$ is shown by the dotted line. The dashed-dotted line indicates the linearized van't Hoff law. (c) Activity coefficient $\gamma_{e}$ used in Eq. (5), for the solid and dashed lines in panel (b), in comparison with the values taken from Ref. 25.

\section{A. Flux and osmotic pressure: CNT membranes}

Under a pressure drop $\Delta p$, the fluxes of ethanol $Q_{e}$ and water $Q_{w}$ are deduced from the linear fit of the time dependent variation of the number of molecules crossing the membrane $\Delta N_{e, w}^{B}(t)$,

$$
Q_{e, w}=\frac{M_{e, w}}{\rho_{e, w} N_{\mathrm{A}}} \frac{\mathrm{d} \Delta N_{e, w}^{B}}{\mathrm{~d} t},
$$

where $M_{e, w}$ and $\rho_{e, w}$ are, respectively, the molar mass and density of ethanol and water, and $N_{\mathrm{A}}$ is the Avogadro constant. The flux $Q_{e}$ of ethanol for a membrane of nanotubes of radius $a_{c}=4.7 \AA$ and length $L=13 \AA$ is reported in Fig. 2(a), for varying applied pressure difference $\Delta p$. The results are plotted for various values of the initial molar fraction of water $x_{w}$, defined as $x_{w}=N_{w}^{B} /\left(N_{w}^{B}+N_{e}^{B}\right)$, with $N_{e, w}^{B}$ being the initial number of molecules in the bottom reservoir.

\section{Permeability}

A first feature of Fig. 2(a) is that the flux of ethanol is found to be linear in the applied pressure, regardless of the concentration in water. From the plot, the hydrodynamic permeance $\mathcal{L}$ of pure ethanol $\left(x_{w}=0\right)$ is extracted, which is defined as the flux per unit area normalized by the pressure drop,

$$
\mathcal{L}=\frac{Q_{e}}{A \Delta p} \approx 1104 \text { liter } /\left(\mathrm{m}^{2} \cdot \mathrm{h} \cdot \mathrm{bar}\right),
$$

where $A$ is the area of the membrane.

This result can be compared to hydrodynamic predictions. Since the channel length is relatively short $\left(L / a_{c} \sim 1\right)$ and the slip length of ethanol inside CNTs is large, ${ }^{27}$ the viscous entrance effect ${ }^{28}$ is expected to dominate the overall dissipation. This effect, which originates in the bending of the streamlines toward the pore, was first discussed by Sampson who calculated the velocity profile flowing through an infinitely thin membrane pierced with circular hole. ${ }^{29}$ In this case, the total flow rate $Q$ is linked to the pressure drop $\Delta p$ through

$$
Q=\frac{a^{3}}{C \eta} \Delta p,
$$

where $a$ is the pore radius (effective radius, see below) and $\eta$ the fluid viscosity. $C$ is a numerical constant, which is $C=3$ for no-slip boundary conditions, but may differ for slipping nanotube surfaces. ${ }^{30}$ Under the present conditions, $C \approx 1.4$ for a CNT with radius $a_{c}=4.7 \AA$ (see Ref. 30 for details). Using this value, Eq. (3) predicts for pure ethanol

$$
\mathcal{L}^{\text {th }}=\frac{a^{3}}{C \eta A} \approx 10^{3} \text { liter } /\left(\mathrm{m}^{2} \cdot \mathrm{h} \cdot \mathrm{bar}\right),
$$

where we used $\eta=1.1 \pm 0.1 \mathrm{mPa} \mathrm{s}$ for the viscosity of ethanol ${ }^{31}$ and $a$ is the effective radius of the tube given by $a \approx a_{c}-2.5 \AA$ (taking into account the steric repulsion at the wall surface). Note that the contribution of the Poiseuille-type dissipation inside the CNT is negligible as compared to the entrance effect computed above, due to the large slip at the CNT surface.

\section{Osmotic pressure}

Beyond the linear dependence of the flux on the pressure, a more unexpected feature of the results in Fig. 2(a) is the existence of an offset in the pressure drop for $x_{w} \neq 0$ : for small pressure drops, the ethanol flux is negative, i.e., directed towards the ethanol-water mixture, and it becomes positive only above a threshold pressure drop. This is the signature of an osmotic pressure expressed by the mixture across the membrane, suggesting that the carbon membrane is semi-permeable to water. This result is surprising becausefor most confinements- the CNT membrane is in general permeable to both water and ethanol when they flow as pure components. ${ }^{13}$ Accordingly the membranes become "selfsemi-permeable" to water due to a preferred adsorption of ethanol in nanoconfinement of the carbon membrane, as compared to the water, see Sec. III A 4.

Let us first explore more quantitatively the osmotic pressure. For a membrane semi-permeable to water, the ethanol flow is expected to be proportional to $\Delta p-\Delta \Pi$ 
where $\Delta \Pi$ is the osmotic pressure due to the difference in water concentration across the membrane. A simple thermodynamic formula for this osmotic pressure of the mixture yields $^{32}$

$$
\Delta \Pi=-\frac{\rho_{e} N_{\mathrm{A}} k_{\mathrm{B}} T}{M_{e}} \sigma \ln \left(\gamma_{e}\left(1-x_{w}\right)\right),
$$

where $k_{\mathrm{B}}$ is the Boltzmann constant and $\gamma_{e}$ is the activity coefficient of ethanol as a function of molar fraction of water $x_{w}$. The so-called reflection coefficient $\sigma$ accounts for the effect of incomplete rejection of the solute (water in our case) through the membrane, ${ }^{33,34}$ which at this stage is assumed to be unity. Note that in the limit of a dilute solution, i.e., $N_{w}^{B} \rightarrow 0$ thus $x_{w} \rightarrow 0$ and $\gamma_{e} \rightarrow 1$, this formula reduces to the van't Hoff law: $\Delta \Pi=k_{\mathrm{B}} T c_{w}$, where $c_{w}$ is the concentration of water defined as the number of molecules per unit volume.

Figure 2(b) shows the measured osmotic pressure $\Delta \Pi$ in comparison with Eq. (5), as a function of the initial value of $c_{w}$ in the bottom reservoir. The osmotic pressure of the MD results is obtained from Fig. 2(a), by measuring the intersection point at $Q_{e}=0$. The relation between concentration $c_{w}$ and molar fraction $x_{w}$ is $c_{w}=x_{w} N_{\mathrm{A}} /\left[M_{e}\left(1-x_{w}\right) / \rho_{e}+M_{w} x_{w} / \rho_{w}\right]$. The values for the activity coefficient $\gamma_{e}$ used for the theoretical comparison of the osmotic pressure-solid line in Fig. 2(b) -are shown in Fig. 2(c). They are found to match very well the experimental values. ${ }^{25}$ Altogether an excellent agreement is found between the theoretical prediction (with $\sigma=1$ ) and the MD results, assessing the semi-permeable character of the present CNT membrane to water in the water-ethanol mixture. The rejection will be studied more quantitatively in Sec. III A 3.

Another observation for the mixture is that the slope of the $Q_{e}$ versus $\Delta p$ curve-i.e., the hydrodynamic permeance to ethanol-appears to slightly decrease for increasing water concentration (with $\sim 10 \%$ variation). This suggests that the accumulated water molecules that appear near the membrane in the steady state provide an additional resistance to ethanol flow. ${ }^{35}$

\section{Water selectivity}

We now explore more exhaustively the water selectivity of the CNT membrane as a function of the pore size. To this end, we consider a water-ethanol mixture with an initial molar fraction of $x_{w}=0.5$, i.e., a 50\%-50\% mixture. The flux of each component is measured across the membrane under a given pressure-drop $\Delta p=400$ bars, and this procedure is repeated for various tube radii $a_{c}$ for the CNT membrane. Results are reported in Fig. 3(a). As expected both fluxes (water and ethanol) increase for increasing tube radius. Note that for the smaller tubes $\left(a_{c}=3.5,3.9\right.$, and $\left.4.3 \AA\right)$, no water molecule is recorded in the top reservoir during the total duration of the simulation, corresponding to $40 \mathrm{~ns}$. In line with the observation in Sec. III A 2, the flux of ethanol is at least one order of magnitude larger than the flux of water.

In order to quantify the efficiency of the separation, we define the rejection coefficient of the membrane as
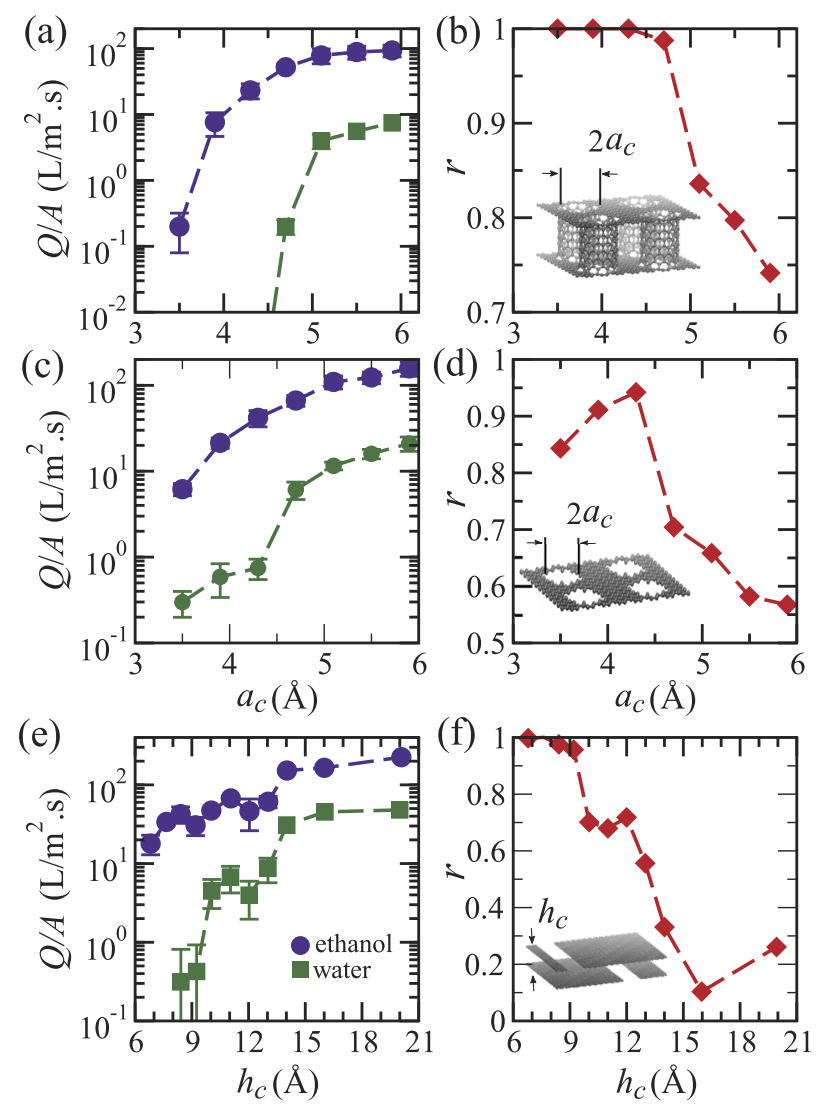

FIG. 3. Flow of ethanol-water mixture across carbon-based membranes of various types. Left: partial flux of ethanol and water per area $Q / A$ for an ethanol-water mixture of $x_{w}=0.5$ flowing through (a) a CNT membrane and (c) a nanoporous graphene sheet, as a function of the pore size $a_{c}$, under an applied pressure of $\Delta p=400$ bars. The corresponding flux through a multilayer graphene membrane with $\Delta p=800$ bars is plotted in panel (e), as a function of the inter-layer distance $h_{c}$. Right: Rejection coefficient $r$ (defined in Eq. (6)) for (b) the CNT membrane, (d) the nanoporous graphene, and (f) the multilayer graphene membrane.

$$
r=1-c \frac{Q_{w}}{Q_{e}},
$$

where $c$ is defined as $c=M_{e} \rho_{w}\left(1-x_{w}\right) / M_{w} \rho_{e} x_{w}$. The prefactor $c$ is such that the rejection coefficient $r$ is equal to 1 for a membrane completely impermeable to water and is equal to 0 for a membrane equally permeable to both ethanol and water. As seen in Fig. 3(b), $r$ is close to 1 for $a_{c} \sim 4.7 \AA$ and jumps down to 0.84 for $a_{c}=5.1 \AA$. Note that $r$ is unity for pore radius below $4.7 \AA$ as the water flux is negligible, predicting excellent separation performance for those radii. The jump of the rejection coefficient between 4.7 and $5.1 \AA$ in radius echoes a previous result for water transport in CNT, in Ref. 36, where we showed that in this radius range, disjoining pressure effects reduce water adsorption in CNT. This entropic effect may add up to the separation while having no effect on ethanol permeability, in good agreement with the present results.

\section{Affinity with the membrane}

We now investigate the (molecular) mechanism underlying the observed "self-semi-permeability." As we show here, the observed self-semi-permeability stems from the high 

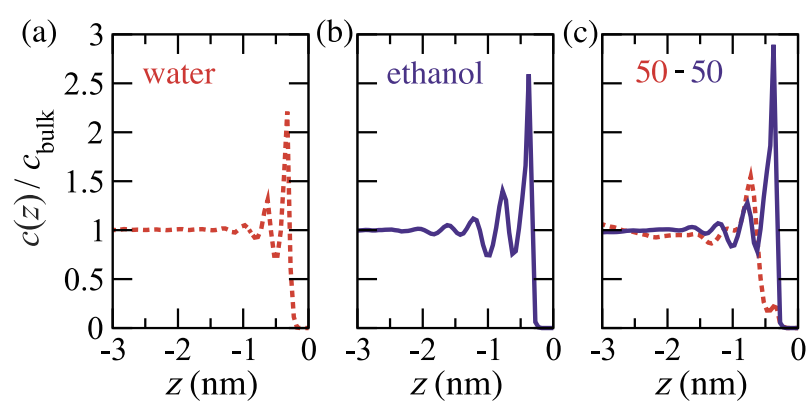

FIG. 4. Concentration profiles of (a) pure water, (b) pure ethanol, and (c) mixture of $50 \%$ water-50\% ethanol, near a graphene sheet located at $z=0$. The profiles are normalized by their respective concentration in the bulk.

affinity between the graphene surface and ethanol molecules, in comparison to the graphene-water interaction. This preferred affinity is highlighted by the detailed concentration profiles of water and ethanol near a graphene surface, as shown in Fig. 4. Both pure ethanol and water liquids show a large absorption near the graphene sheet, with the presence of a peak in the density profile (Figs. 4(a) and 4(b)). However in the case of a mixture, a higher affinity for the ethanol molecules is clearly observed in Fig. 4(c), with a strong peak of ethanol in the first layer near the graphene sheet at $z=0$, while most of the water molecules are displaced further away from the carbon surface. This preferred affinity of ethanol allows to rationalize the preferred adsorption of ethanol in nanoconfining structure and the effective rejection of water molecules, as we observe above in the membranes. More into the details, water is present in the second adsorption layer $(\geq 0.5 \mathrm{~nm}$ away from the carbon surface). This suggests that the self-semi-permeability requires confinement to be smaller than (roughly) two molecular layers. This is in agreement with the decrease of the rejection coefficient in this range of confinement as observed in Fig. 3 for the three membranes considered here.

Furthermore, our findings are in agreement with experiments reported in Ref. 37, showing the limited insertion of water into graphite oxide in the presence of alcohol (methanol) in a mixture. This points altogether to a robust physical mechanism and to the possibility of the separation using the GO membranes. Beyond the consequences on the osmotic behavior discussed here, this suggests a rich behavior of the static and structure properties of confined mixtures, as pointed out in Ref. 38.

Finally, in order to assess that the effect is related to the preferred adsorption of ethanol, we further checked the influence of the solvent-carbon interaction strength. We performed additional simulations of water selectivity with three different force fields (using OPLS-AA ${ }^{39}$ instead of OPLS-UA for ethanol, using SPC/E model ${ }^{40}$ instead of TIP4P/2005 model for water, and using different LennardJones parameters for carbon atoms ${ }^{41}$ ). These simulations gave qualitatively similar results as those shown here, with only slight quantitative changes. This therefore supports the mechanism discussed in this section and does confirm the robustness of the self-semi-permeability effect.

\section{B. Generalization to nanoporous and multilayer graphene membranes}

Beyond the CNT membrane, the above procedure was applied to the various carbon membranes under consideration: a graphene sheet pierced with circular pores, reminiscent of the developing nanoporous graphene membranes, ${ }^{42}$ and multilayer graphene membranes, as depicted in Figs. 1(c) and 1(d). The latter geometry is considered as a model of the porous structure of the graphene-oxide (GO) membranes. ${ }^{26,43-47}$ Overall the very same features are exhibited by all considered carbon membranes, namely, the following:

(i) The membranes become "self-semi-permeable" to water in the presence of water-ethanol mixtures, although both pure components pass freely through them; this behavior is highlighted in Fig. 5 for multilayer GO-like graphene membranes.

(ii) This semi-permeable character manifests itself in the expression of an osmotic pressure, obeying the van't Hoff type expression, see Fig. 2(b) for multilayer graphene membranes and CNT membranes.

(iii) A size dependent water selectivity is measured, as highlighted in Fig. 3, confirming semi-permeability for the smallest pore size.

Overall an identical behavior for various confinement geometries points to a robust and generic mechanism. In line with the findings for the CNT membrane, the
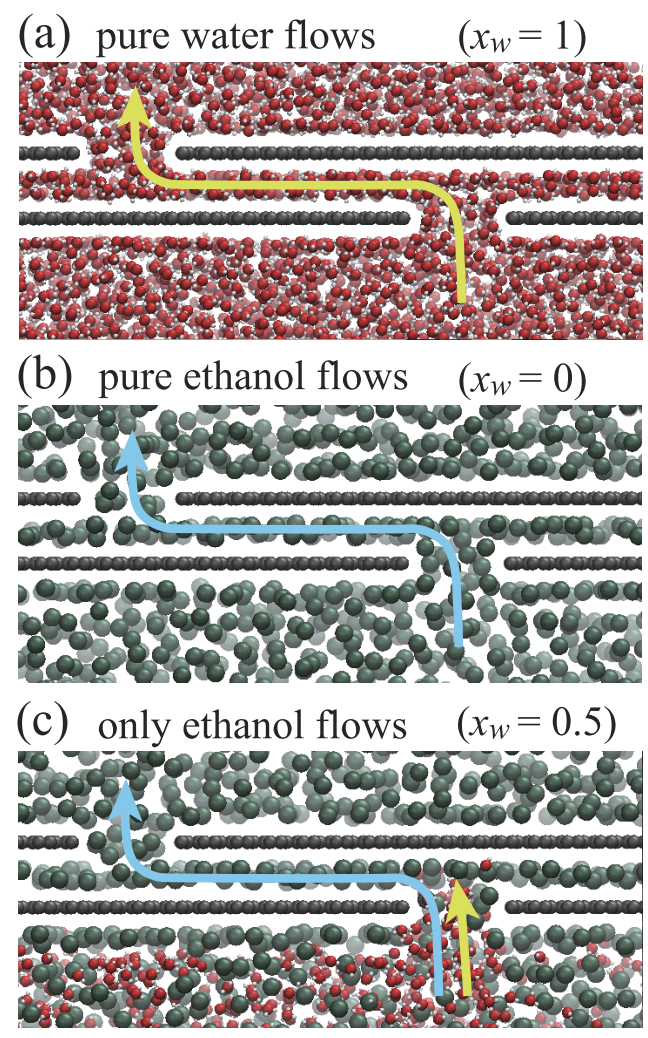

FIG. 5. Snapshots of flows through the multilayer graphene membrane with $h_{c}=8.4 \AA$, for (a) $x_{w}=1$ (with the top reservoir filled with water), (b) $x_{w}=0$, and (c) $x_{w}=0.5$. The ethanol molecule is represented by a particle at the position of $\mathrm{CH}_{2}$. 
efficient separation of the carbon membranes originates in the high affinity between carbon atoms and ethanol molecules, which leads to preferred carbon adsorption in the nanoconfinement as compared to water. Accordingly, the effect of separation persists regardless of the details and geometry of the membrane, as long as the latter is made of carbon atom and presents small pores (typically with a diameter $\leq 1 \mathrm{~nm})$.

The osmotic pressure $\Delta \Pi$ for the multilayer GO-like graphene membranes is plotted in Fig. 2(b). For the case of $h_{c}=8.4 \AA$, the osmotic pressure is again in good agreement with the thermodynamic prediction given in Eq. (5), which confirms the semi-permeable character of this membrane. On the other hand, smaller values of $\Delta \Pi$ are obtained for the GO-like membrane of $h_{c}=12 \AA$. This implies that the rejection of water molecules is incomplete at this inter-layer distance. Indeed, as shown by the dashed line in Fig. 2(b), the reduction of osmotic pressure is still captured by Eq. (5) with employing the value of the reflection coefficient smaller than unity, i.e., $\sigma=0.7$.

As shown in Fig. 3, the three carbon membranes exhibit a similar rejection behavior, with a rejection coefficient going from unity down for small pores to small rejection values as the typical size of the pore (CNT diameter, pore size in graphene, or inter-layer gap for multilayer GO) bypasses a few angtröms. The range of rejection matches for all different membranes: typically the water exhibits high rejection $(r \approx 1)$ for pore diameter below $10 \AA$ for both CNTs and graphene pores (with a better performance for CNTs) and for inter-layer gaps below $\sim 10 \AA$ for multilayer GO. We note already at this stage that while it is difficult to fabricate macroscopic membranes of CNT and nanoporous graphene with such specificities on the pore diameters, inter-layer gaps in this range are quite common for macroscopic GO membranes. ${ }^{48}$ This is actually a very interesting feature for practical up-scaling of the process.

We also explored the influence of increasing the number of layers in the multilayer GO membrane. As one may expect, we found that selectivity increased with this number. For the inter-layer distance $h_{c}=12 \AA$, the rejection coefficient reaches almost unity $(r>0.97)$ with five-layer membrane, with the flux of ethanol remaining in the same order of magnitude as the two-layer membrane. Furthermore, we note as a side remark that the measured values of flux for ethanol are in the typical range of permeability estimate, ${ }^{26}$ which compares well with the experimental results for GO membranes. ${ }^{44,45}$

\section{DISCUSSION}

Altogether, our results demonstrate that carbon-based membranes can be used to separate very efficiently ethanol from water, thereby suggesting their potential for membranebased separation of these two elements. ${ }^{49}$ In a very counterintuitive way, these carbon-based membranes are shown to be generally permeable to both liquids when considered as pure components but become semi-permeable to water for water-ethanol mixtures, as highlighted in Fig. 5. This effective selectivity takes its origin in the high affinity of ethanol to these carbon membranes as compared to water, an effect which is strongly enhanced for sufficiently small pore dimensions. This separation mechanism is therefore robust, simple, and quite independent of the geometry considered, as highlighted for the various types of carbon-based membranes considered in the present study. This effect leads to a rejection coefficient of water close to one for carbon membranes with subnanometric pores, when in the presence of water-ethanol mixtures. We further expect this separation process to apply not only to the ethanol-water mixture but also to any similar molecule, such as methanol. ${ }^{37}$ In addition, one may expect that the mechanisms behind the self-semi-permeability should persist to some extent in other hydrophobic (e.g., polymeric) nanoporous membranes. ${ }^{50}$

In order to highlight the potential of the membrane-based separation, let us quantify the energetics of the process. To put numbers, we consider a multilayer graphene membrane as a model system. Similar results are obtained with the two other types of membranes, but this choice is particularly relevant because such graphene-oxide-like membranes are prone to easy scale-up. To fix ideas, we consider an multilayer carbon membrane with an inter-layer distance of $h_{c}=8.4 \AA$. As shown in Fig. 3, this leads to nearly perfect water rejection, $r \simeq 1$. A $50 \%-50 \%$ water-ethanol mixture corresponds to an osmotic pressure $\Delta \Pi \approx 200$ bars, see Fig. 2 . The ethanol flux under an applied pressure of $\Delta p=800$ bars is found to be $Q / A \sim 43$ liter $/ \mathrm{m}^{2} \cdot \mathrm{s}$ (see Fig. 3), while the water flux is negligible. Assuming that the flux is proportional to $\Delta p-\Delta \Pi$, (see Sec. III A 2), an ethanol flow rate of $Q_{e} \sim 3.5$ liter/s for a $1 \mathrm{~m}^{2}$ membrane will be driven under an applied pressure of $\Delta p=250$ bars. The corresponding required power is accordingly $\mathcal{P}=Q_{e} \times \Delta p \sim 88 \mathrm{~kW}$. The cost for separating, say, 1 liter of water-ethanol, is then $\sim 25 \mathrm{~kJ}$. This energy cost is to be compared to the thermodynamic limit for the energy cost of separating such a mixture, which is $17 \mathrm{~kJ}$ (see, e.g., Ref. 51). Furthermore the energy required for boiling in a typical azeotropic distillation process, based on an extrapolation of reported value, ${ }^{52}$ is approximately $3 \mathrm{MJ}$, which is two orders of magnitude larger than that estimated for the carbon multilayer membrane. Similar numbers are expected for the other CNT and nanoporous graphene membranes.

These predictions are accordingly highly attractive as an alternative solution for water-ethanol separation. More specifically, the results for the CNT and nanoporous membranes may certainly suggest high expectations, and the fabrication of carbon nanotube membranes, ${ }^{53,54}$ carbon nanotube-mixed matrix membranes, ${ }^{10}$ and ultra-thin nanoporous graphene membranes, ${ }^{55}$ has been reported in the recent literature. But the practical scaling-up of such membranes up to square meters still remains a technological challenge. In contrast, grapheneoxide membranes, which are intrinsically large scale and easy to fabricate, ${ }^{56-58}$ make such layered carbon membranes a far more plausible candidate to highlight the present effect. We believe that the potentially huge reduction in energy cost offered by the present membrane-based process makes it a serious candidate for water-ethanol separation at large scales. 


\section{ACKNOWLEDGMENTS}

This research was supported by the European Research Council program Micromegas project. It was also granted access to the HPC resources of MesoPSL financed by the Region Ile de France and the project Equip@Meso (Reference No. ANR-10-EQPX-29-01) of the programme Investissements d'Avenir supervised by the Agence Nationale de la Recherche (ANR).

${ }^{1}$ M. Balat and H. Balat, "Recent trends in global production and utilization of bio-ethanol fuel," Appl. Energy 86, 2273-2282 (2009).

${ }^{2}$ B. Hahn-Hägerdal, M. Galbe, M. F. Gorwa-Grauslund, G. Lidén, and G. Zacchi, "Bio-ethanol-the fuel of tomorrow from the residues of today," Trends Biotechnol. 24, 549-556 (2006).

${ }^{3}$ H.-J. Huang, S. Ramaswamy, U. W. Tschirner, and B. V. Ramarao, "A review of separation technologies in current and future biorefineries," Sep. Purif. Technol. 62, 1-21 (2008)

${ }^{4}$ M. H. V. Mulder, J. O. Hendrickman, H. Hegeman, and C. A. Smolders, "Ethanol-water separation by pervaporation," J. Membr. Sci. 16, 269-284 (1983).

${ }^{5}$ T. Sano, H. Yanagishita, Y. Kiyozumi, F. Mizukami, and K. Haraya, "Separation of ethanol/water mixture by silicalite membrane on pervaporation," J. Membr. Sci. 95, 221-228 (1994).

${ }^{6}$ M. H. V. Mulder, A. C. M. Franken, and C. A. Smolders, "On the mechanism of separation of ethanol/water mixtures by pervaporation. II. Experimental concentration profiles," J. Membr. Sci. 23, 41-58 (1985).

${ }^{7}$ M. Nomura, T. Yamaguchi, and S.-I. Nakao, "Ethanol/water transport through silicalite membranes," J. Membr. Sci. 144, 161-171 (1998).

${ }^{8}$ R. Semiat, "Energy issues in desalination processes," Environ. Sci. Technol. 42, 8193-8201 (2008).

${ }^{9}$ S. S. Sablani, M. F. A. Goosen, R. Al-Belushi, and M. Wilf, "Concentration polarization in ultrafiltration and reverse osmosis: A critical review," Desalination 141, 269-289 (2001).

${ }^{10}$ A. F. Ismail, P. S. Goh, S. M. Sanip, and M. Aziz, "Transport and separation properties of carbon nanotube-mixed matrix membrane," Sep. Purif. Technol. 70, 12-26 (2009).

${ }^{11}$ K. P. Lee, T. C. Arnot, and D. Mattia, "A review of reverse osmosis membrane materials for desalination-development to date and future potential," J. Membr. Sci. 370, 1-22 (2011).

${ }^{12} \mathrm{C}$. Fritzmann, J. Löwenberg, T. Wintgens, and T. Melin, "State-of-the-art of reverse osmosis desalination," Desalination 216, 1-76 (2007).

${ }^{13}$ Note that we find that all carbon membranes are permeable to pure water, except the CNT membrane for a specific range of pore radii $a_{c} \in[4.3-4.7] \AA$ A. See Ref. 36.

${ }^{14}$ S. Plimpton, "Fast parallel algorithms for short-range molecular dynamics," J. Comput. Phys. 117, 1-19 (1995).

${ }^{15}$ See http://lammps.sandia.gov for the code.

${ }^{16}$ J. L. F. Abascal and C. Vega, "A general purpose model for the condensed phases of water: TIP4P/2005," J. Chem. Phys. 123, 234505 (2005).

${ }^{17}$ W. L. Jorgensen, J. D. Madura, and C. J. Swenson, "Optimized intermolecular potential functions for liquid hydrocarbons," J. Am. Chem. Soc. 106, 6638-6646 (1984).

${ }^{18} \mathrm{~W}$. L. Jorgensen, "Optimized intermolecular potential functions for liquid alcohols," J. Phys. Chem. 90, 1276-1284 (1986).

${ }^{19}$ W. D. Cornell, P. Cieplak, C. I. Bayly, I. R. Gould, K. M. Merz, D. M. Ferguson, D. C. Spellmeyer, T. Fox, J. W. Caldwell, and P. A. Kollman, "A second generation force field for the simulation of proteins, nucleic acids, and organic molecules," J. Am. Chem. Soc. 117, 5179-5197 (1995).

${ }^{20} \mathrm{~A}$. Alexiadis and S. Kassinos, "Molecular simulation of water in carbon nanotubes," Chem. Rev. 108, 5014-5034 (2008).

${ }^{21}$ J. A. Thomas and A. J. H. McGaughey, "Water flow in carbon nanotubes: Transition to subcontinuum transport," Phys. Rev. Lett. 102, 184502 (2009).

${ }^{22}$ T. Werder, J. H. Walther, R. L. Jaffe, T. Halicioglu, and P. Koumoutsakos, "On the water-carbon interaction for use in molecular dynamics simulations of graphite and carbon nanotubes," J. Phys. Chem. B 107, 1345-1352 (2003).

${ }^{23}$ H. J. C. Berendsen, J. P. M. Postma, W. F. van Gunsteren, A. DiNola, and J. R. Haak, "Molecular dynamics with coupling to an external bath," J. Chem. Phys. 81, 3684-3690 (1984).

${ }^{24} \mathrm{M}$. Thomas and B. Corry, "Thermostat choice significantly influences water flow rates in molecular dynamics studies of carbon nanotubes," Microfluid. Nanofluid. 18, 41-47 (2015).
${ }^{25}$ D. W. Green and R. H. Perry, Perry's Chemical Engineers' Handbook, 8th ed. (McGraw-Hill, 2007).

${ }^{26} \mathrm{H}$. Yoshida and L. Bocquet, "Labyrinthine water flow across multilayer graphene-based membranes: Molecular dynamics versus continuum predictions," J. Chem. Phys. 144, 234701 (2016).

${ }^{27}$ K. Falk, F. Sedlmeier, L. Joly, R. R. Netz, and L. Bocquet, "Ultralow liquid/solid friction in carbon nanotubes: Comprehensive theory for alcohols, alkanes, OMCTS, and water," Langmuir 28, 14261-14272 (2012).

${ }^{28}$ T. B. Sisan and S. Lichter, "The end of nanochannels," Microfluid. Nanofluid. 11, 787-791 (2011).

${ }^{29}$ R. A. Sampson, "On Stokes's current function," Philos. Trans. R. Soc., A 182, 449-518 (1891).

${ }^{30}$ S. Gravelle, L. Joly, C. Ybert, and L. Bocquet, "Large permeabilities of hourglass nanopores: From hydrodynamics to single file transport," J. Chem. Phys. 141, 18C526 (2014).

${ }^{31}$ G. Guevara-Carrion, C. Nieto-Draghi, J. Vrabec, and H. Hasse, "Prediction of transport properties by molecular simulation: Methanol and ethanol and their mixture," J. Phys. Chem. B 112, 16664-16674 (2008).

${ }^{32}$ I. M. Klotz and R. M. Rosenberg, Chemical Thermodynamics Basic Concepts and Methods, 7th ed. (John Wiley \& Sons, Inc., 2008).

${ }^{33}$ A. J. Staverman, "The theory of measurement of osmotic pressure," Recl. Trav. Chim. Pays-Bas 70, 344-352 (1951).

${ }^{34}$ S. J. Fritz, "Ideality of clay membranes in osmotic processes: A review," Clays Clay Miner. 34, 214-223 (1986).

${ }^{35}$ J. G. Wijmans, S. Nakao, J. W. A. Van Den Berg, F. R. Troelstra, and C. A. Smolders, "Hydrodynamic resistance of concentration polarization boundary layers in ultrafiltration,” J. Membr. Sci. 22, 117-135 (1985).

${ }^{36}$ S. Gravelle, C. Ybert, L. Bocquet, and L. Joly, "Anomalous capillary filling and wettability reversal in nanochannels," Phys. Rev. E 93, 033123 (2016).

${ }^{37}$ S. You, J. Yu, B. Sundqvist, L. A. Belyaeva, N. V. Avramenko, M. V. Korobov, and A. V. Talyzin, "Selective intercalation of graphite oxide by methanol in water/methanol mixtures," J. Phys. Chem. C 117, 1963-1968 (2013).

${ }^{38} \mathrm{M}$. Zhao and X. Yang, "Segregation structures and miscellaneous diffusions for ethanol/water mixtures in graphene-based nanoscale pores," J. Phys. Chem. C 119, 21664-21673 (2015).

${ }^{39}$ W. L. Jorgensen, D. S. Maxwell, and J. Tirado-Rives, "Development and testing of the OPLS all-atom force field on conformational energetics and properties of organic liquids," J. Am. Chem. Soc. 118, 11225-11236 (1996).

${ }^{40}$ H. J. C. Berendsen, J. R. Grigera, and T. P. Straatsma, "The missing term in effective pair potentials,” J. Phys. Chem. 91, 6269-6271 (1987).

${ }^{41}$ A. Striolo, P. K. Naicker, A. A. Chialvo, P. T. Cummings, and K. E. Gubbins, "Simulated water adsorption isotherms in hydrophilic and hydrophobic cylindrical nanopores," Adsorption 11, 397-401 (2005).

${ }^{42}$ D.-E. Jiang, V. R. Cooper, and S. Dai, "Porous graphene as the ultimate membrane for gas separation," Nano Lett. 9, 4019-4024 (2009).

${ }^{43}$ R. R. Nair, H. A. Wu, P. N. Jayaram, I. V. Grigorieva, and A. K. Geim, "Unimpeded permeation of water through helium-leak-tight graphene-based membranes," Science 335, 442-444 (2012).

${ }^{44}$ S. Xia, M. Ni, T. Zhu, Y. Zhao, and N. Li, "Ultrathin graphene oxide nanosheet membranes with various $\mathrm{d}$-spacing assembled using the pressureassisted filtration method for removing natural organic matter," Desalination 371, 78-87 (2015).

${ }^{45} \mathrm{M}$. Hu and B. Mi, "Enabling graphene oxide nanosheets as water separation membranes," Environ. Sci. Technol. 47, 3715-3723 (2013).

${ }^{46}$ S. Ban, J. Xie, Y. Wang, B. Jing, B. Liu, and H. Zhou, "Insight into the nanoscale mechanism of rapid $\mathrm{H}_{2} \mathrm{O}$ transport within graphene oxide membrane: The impact of oxygen functional group clustering," ACS Appl. Mater. Interfaces 8, 321-332 (2016).

${ }^{47}$ A. Akbari, P. Sheath, S. T. Martin, D. B. Shinde, M. Shaibani, P. C. Banerjee, R. Tkacz, D. Bhattacharyya, and M. Majumder, "Large-area graphene-based nanofiltration membranes by shear alignment of discotic nematic liquid crystals of graphene oxide," Nat. Commun. 7, 10891 (2016).

${ }^{48}$ X. Yang, C. Cheng, Y. Wang, L. Qiu, and D. Li, "Liquid-mediated dense integration of graphene materials for compact capacitive energy storage," Science 341, 534-537 (2013).

${ }^{49}$ J. R. Werber, A. Deshmukh, and M. Elimelech, "The critical need for increased selectivity, not increased water permeability, for desalination membranes," Environ. Sci. Technol. Lett. 3, 112-120 (2016).

${ }^{50}$ D. Surblys, Y. Yamaguchi, K. Kuroda, M. Kagawa, T. Nakajima, and H. Fujimura, "Molecular dynamics analysis on wetting and interfacial properties of water-alcohol mixture droplets on a solid surface," J. Chem. Phys. 140, 034505 (2014).

${ }^{51}$ R. Agrawal and D. M. Herron, "Optimal thermodynamic feed conditions for distillation of ideal binary mixtures,” AIChE J. 43, 2984-2996 (1997). 
${ }^{52}$ G. J. Prokopakis and W. D. Seider, "Dynamic simulation of azeotropic distillation towers," AIChE J. 29, 1017-1029 (1983).

${ }^{53}$ J. K. Holt, H. G. Park, Y. Wang, M. Stadermann, A. B. Artyukhin, C. P. Grigoropoulos, A. Noy, and O. Bakajin, "Fast mass transport through sub2-nanometer carbon nanotubes," Science 312, 1034-1037 (2006).

${ }^{54}$ M. Majumder, N. Chopra, R. Andrews, and B. J. Hinds, "Enhanced flow in carbon nanotubes," Nature 438, 44 (2005).

${ }^{55}$ T. J. Booth, P. Blake, R. R. Nair, D. Jiang, E. W. Hill, U. Bangert, A. Bleloch, M. Gass, K. S. Novoselov, M. I. Katsnelson, and A. K. Geim, "Macroscopic graphene membranes and their extraordinary stiffness," Nano Lett. 8, 2442-2446 (2008).
${ }^{56}$ D. A. Dikin, S. Stankovich, E. J. Zimney, R. D. Piner, G. H. B. Dommett, G. Evmenenko, S. T. Nguyen, and R. S. Ruoff, "Preparation and characterization of graphene oxide paper," Nature 448, 457-460 (2007).

${ }^{57}$ H. Li, Z. Song, X. Zhang, Y. Huang, S. Li, Y. Mao, H. J. Ploehn, Y. Bao, and $\mathrm{M}$. Yu, "Ultrathin, molecular-sieving graphene oxide membranes for selective hydrogen separation," Science 342, 95-98 (2013).

${ }^{58}$ H. W. Kim, H. W. Yoon, S.-M. Yoon, B. M. Yoo, B. K. Ahn, Y. H. Cho, H. J. Shin, H. Yang, U. Paik, S. Kwon, J.-Y. Choi, and H. B. Park, "Selective gas transport through few-layered graphene and graphene oxide membranes," Science 342, 91-95 (2013). 